\title{
Quantitative assessment of nipple perfusion with laser- assisted indocyanine green imaging in nipple-sparing mastectomy with breast reconstruction
}

Jaewoo Kim¹, Il-Kug Kim², Ung Sik Jin ${ }^{1}$, Hak Chang ${ }^{1}$

${ }^{1}$ Department of Plastic and Reconstructive Surgery, Seoul National University College of Medicine, Seoul; ${ }^{2}$ Department of Plastic and Reconstructive Surgery, Yeungnam University College of Medicine, Daegu, Korea

This article was presented at the PRS Korea 2018 on November 9-11, 2018 in Seoul, Korea.
Background It remains unknown whether perfusion mapping using the SPY system can predict mastectomy skin flap necrosis in each type of breast surgery. We analyzed intraoperative indocyanine green (ICG) angiography images of breast cancer patients who underwent nipple-sparing mastectomy (NSM) with implant-based breast reconstruction, and evaluated the perfusion of the nipple-areolar complex (NAC) with the SPY Elite system to predict NAC necrosis with a single quantitative value.

Methods We analyzed nipple perfusion in 30 patients from October 2016 to November 2018. After NSM, ICG injection and SPY angiography were performed to characterize NAC perfusion before immediate reconstruction. The nipple perfusion rate was measured by analyzing fluorescence at the central point of the nipple, and the presence of NAC necrosis was evaluated at 5 days and 1 month postoperatively.

Results Three of the 30 patients developed NAC necrosis that secondarily healed within 1 month, and five developed NAC necrosis and underwent surgical debridement within 1 month. Seven of eight patients with a perfusion rate $<13 \%$ developed NAC necrosis, and all four patients with a perfusion rate $<10 \%$ needed partial surgical debridement or total NAC excision.

Conclusions The nipple perfusion rate could be useful for predicting NAC necrosis before immediate reconstruction. For patients at a high risk for NAC necrosis, tissue expander insertion rather than a direct-to-implant procedure may be considered, and close followup with thorough wound management should be done to reduce complications.

Keywords Mastectomy / Indocyanine green / Nipple / Necrosis

\section{INTRODUCTION}

Breast implant insertion after mastectomy can result in various complications, such as infection, implant exposure, implant rup-

Received: Dec 19, 2018 Revised: Dec 27, 2018 Accepted: Dec 27, 2018 Correspondence: Hak Chang Department of Plastic and Reconstructive Surgery, Seoul National University College of Medicine, 101 Daehak-ro, Jongno-gu, Seoul 03080, Korea

Tel: +82-2-2072-3086, Fax: +82-2-747-5130, E-mail: hchang@snu.ac.kr

Copyright @ 2019 The Korean Society for Aesthetic Plastic Surgery.

This is an Open Access article distributed under the terms of the Creative Commons Attribution Non-Commercial License (http://creativecommons.org/licenses/by-nc/4.0/) which permits unrestricted non-commercial use, distribution, and reproduction in any medium, provided the original work is properly cited. $\quad w w w . e-a a p s . o r g$ ture, or capsular contracture. The viability of the mastectomy skin flap is an important factor for a successful breast reconstruction. To evaluate the viability of the mastectomy skin flap, the SPY Intraoperative Perfusion Assessment System (Novadaq Technologies, Toronto, ON, Canada) can be utilized for intraoperative perfusion mapping. This system utilizes a laser diode array, and a chargecoupled device camera can be configured to capture image sequences. Images are viewed on a high-definition monitor in real time, allowing for immediate evaluation in the operating room. A companion post-processing software product (SPY-Q Analysis Toolkit) provides additional viewing, comparison, and analysis tools, including algorithms for measuring fluorescence intensity [1]. After the intravenous injection of indocyanine green (ICG) dye, the SPY device detects the signal of the dye. 
In 2010, Komorowska-Timek and Gurtner [2] demonstrated that the SPY system could be used to evaluate the perfusion of mastectomy skin flaps and proved that intraoperative perfusion mapping with laser-assisted ICG imaging could predict and prevent complications in immediate breast reconstruction. ICG images from the SPY device showed immediate postoperative decreased perfusion of the mastectomy skin flap in patients who eventually experienced skin flap necrosis as a complication, and through a comparative study of intraoperative interventions based on ICG images, it was shown that the SPY system reduced the incidence of necrosis-related complications. Since then, ICG angiography has gained popularity in breast reconstruction for its ability to assess mastectomy skin and tissue flap viability. In recent article on outcomes in 2018, Chattha et al. [3] identified breast reconstructions performed with or without ICG angiography in 2012 to 2014, and found that ICG angiography was performed in approximately $3.0 \%$ of breast reconstructions; its usage increased over time, and a significant increase in the odds of debridement was associated with its use. In this regard, Mattison et al. [4] questioned the benefit of using the SPY device and claimed that intraoperative ICG angiography was conservative in its estimation of viability and, if followed, may result in a more aggressive resection than the area deemed viable by the operating surgeon.

Whether SPY perfusion mapping can predict mastectomy skin flap necrosis for each type of breast surgery, or when the target is narrowed to a specific location such as the nipple-areolar complex (NAC) or incision site, which are special concerns regarding mastectomy skin flap viability, remains unknown. Recently, nipplesparing mastectomy (NSM) has gained acceptance in the field of breast oncology, because the procedure appears to be oncologically safe with a low risk of cancer recurrence. Thus, the number of patients undergoing NSM has increased due to its cosmetic merits of preserving the native skin and nipple of the breast, and its strength of enabling immediate reconstruction, which can be performed following direct-to-implant breast reconstruction to create a natural appearance of the breast in only a single surgical procedure [5]. Moreover, in NSM, the final survival of the NAC without any necrosis is a key factor for surgical success because NAC necrosis is a relatively common complication (with rates of 3\%-37\%, depending on the study), and it may delay postoperative oncologic therapy and deform the appearance of the breast, resulting in emotional stress [4]. In 2014, Wapnir et al. [6] compared imaging of the nipple perfusion pattern before and after NSM to analyze its relationship with ischemic complications, and concluded that imaging NAC and skin perfusion during NSM was a useful adjunctive tool with the potential to direct the placement of mastectomy incisions and to minimize ischemic complications.

In light of these issues, we analyzed intraoperative ICG angiography images of breast cancer patients who underwent NSM with implant-based breast reconstruction, and evaluated the NAC per- fusion using the SPY system to predict NAC necrosis. Moreover, we tried to predict NAC necrosis with a single quantitative value calculated through a quantitative assessment of nipple perfusion from the ICG image, using the SPY-Q software.

\section{METHODS}

This was a retrospective study of breast cancer patients who underwent NSM and immediate implant-based breast reconstruction at a single institution from October 2016 to November 2018. NSM and immediate reconstruction were offered to patients whose tumors did not involve the skin or NAC. NSM with sentinel lymph node biopsy or axillary lymph node dissection was performed by a single breast surgeon. A lateral incision was made for all patients, circumferential dissection was performed along the subcutaneous plane, and the subareolar and subnipple plane was dissected sharply with routine nipple margin surgical frozen biopsy.

Postmastectomy nipple perfusion was evaluated using the SPY imaging system before immediate reconstruction. Before each evaluation of perfusion, $25 \mathrm{mg}$ of powder ICG dye was dissolved in $5 \mathrm{~mL}$ of saline, and $3 \mathrm{~mL}$ of this solution was administered by the anesthesia team into a peripheral intravenous line followed by a 10 -mL saline flush. Video recording began immediately after injection and continued over 3 minutes. Blood flow in the mastectomy skin flap, including the nipple, was represented as fluorescence or a whitish color on the infrared camera screen, whereas areas with no filling were shown in dark gray or black. Relative nipple perfusion was measured by analyzing the fluorescence at the center point of the nipple at the time of the plateau phase, which is after the arterial and before the venous phase of ICG injection, using the SPY software (Fig. 1). Then, the measured rate was calibrated on a $0-100$ scale, by converting the highest rate in the image to $100 \%$, and converting other rates proportionally.

All patients underwent immediate reconstruction performed by two plastic surgeons (HC and USJ), involving either a direct-to-implant or tissue expander insertion procedure. The selection of an expander or implant was based on the plastic surgeon's experience, considering the mastectomy skin flap pliability and breast width, height, projection, and ptosis grade. The decision was not changed intraoperatively based on the SPY imaging. To make a pocket for the selected expander or implant and whether to use acellular dermal matrix (ADM) was left to the plastic surgeon's discretion. After insertion of the expander or implant, the wound was repaired by layers with two drain insertions. NAC necrosis was evaluated at 5 days after the operation during hospitalization and at 1 month after operation at the outpatient clinic by the plastic surgeon. Cases of NAC necrosis were classified into those that did or did not require revision.

Demographic data of the patients, including age, body mass index (BMI), ethnicity, smoking history, hypertension, diabetes, oth- 

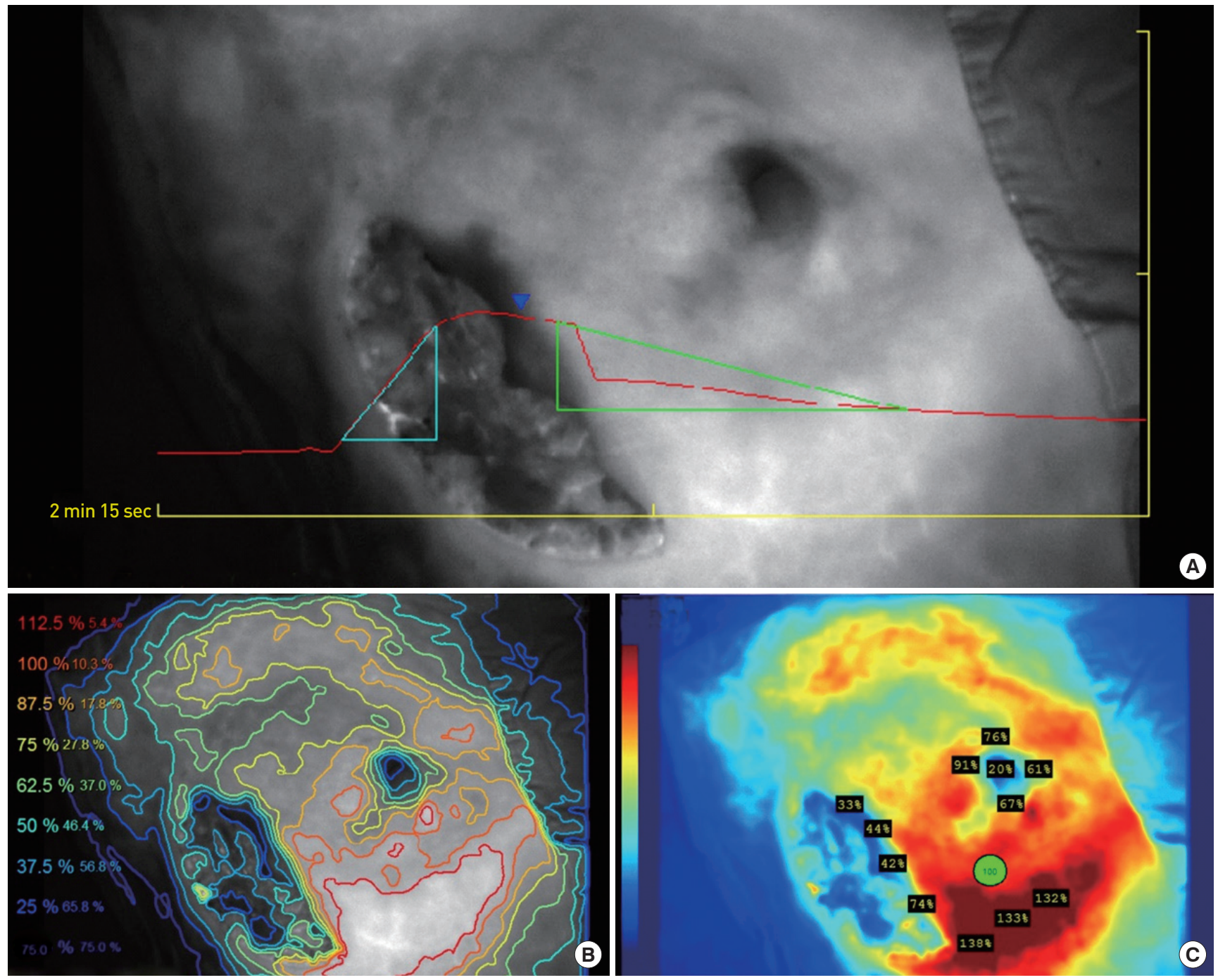

Fig. 1. SPY angiography and relative nipple perfusion. Recorded video file of a sample case. (A) The relative perfusion map image was selected at the time of the plateau phase, which is after the arterial and before the venous phase of the indocyanine green injection. (B) A selected relative perfusion map is shown with contour lines of the same perfusion rates. (C) The image was converted to a color image and the perfusion rate at the center point of the nipple was measured.

er vascular disease, neoadjuvant chemotherapy history, and previous breast surgery history, were collected from the hospital medical record. Patients who later underwent NAC excision owing to positive findings from the nipple margin biopsy and those with previous cosmetic breast surgery history were excluded.

A qualitative analysis for special findings and a quantitative analysis for intergroup comparisons were performed. Statistical analysis of the results was conducted using the Fisher exact test, and the significance level was set at $\mathrm{P}<0.05$. All statistical analyses were performed using SPSS version 25 (IBM Corp., Armonk, NY, USA).

The informed consent was waived, and this investigation was approved by the Institutional Review Board of Seoul National University (IRB No. 1710-065-894).

\section{RESULTS}

All patients were Korean and had a mean age of 44.5 years (range, 26-60 years) and a mean BMI of $22.74 \mathrm{~kg} / \mathrm{m}^{2}$ (range, $18.38-32.24$ $\mathrm{kg} / \mathrm{m}^{2}$ ). Two patients had neoadjuvant chemotherapy before surgery, and no patient underwent postoperative radiation therapy within 1 month. All NSMs were performed using a lateral incision. Mentor expanders (Mentor Worldwide LLC, Irvine, CA, USA) were used for all patients who received an expander, and among the 25 direct-to-implant cases, 11 implants were manufactured by Mentor, 10 by Bellagel (HansBiomed Corp., Seoul, Korea), and three by Allergan (Allergan plc, Dublin, Ireland) with sizes appropriate for each patient. Twenty-three patients had ADM, whereas seven patients had no ADM. One patient had hypertension and 
Table 1. Patient demographics

\begin{tabular}{|c|c|}
\hline Patient factor & Value \\
\hline Age (yr) & $44.47(26-60)$ \\
\hline BMI $\left(\mathrm{kg} / \mathrm{m}^{2}\right)$ & $22.74(18.54-32.24)$ \\
\hline \multicolumn{2}{|l|}{ Laterality } \\
\hline Right breast & $16 / 28(57.1)$ \\
\hline Left breast & $10 / 28(35.7)$ \\
\hline Bilateral breasts & $2 / 28(7.14)$ \\
\hline \multicolumn{2}{|l|}{ Purpose of surgery } \\
\hline Oncologic mastectomy & $27 / 30(90)$ \\
\hline Prophylactic mastectomy & $3 / 30(10)$ \\
\hline \multicolumn{2}{|l|}{ Underlying comorbidities } \\
\hline Active smoking & 0/30 (0) \\
\hline Obesity (BMI > 25 kg/m²) & $5 / 30(17)$ \\
\hline Hypertension & $1 / 30(3)$ \\
\hline Diabetes mellitus & $1 / 30$ (3) \\
\hline \multicolumn{2}{|l|}{ Previous treatment } \\
\hline Preoperative chemotherapy & $2 / 30(7)$ \\
\hline Preoperative radiation therapy & 0/30 (0) \\
\hline \multicolumn{2}{|l|}{ Operation type } \\
\hline Expander & $5 / 30$ (17) \\
\hline Direct-to-implant & 25/30 (83) \\
\hline \multicolumn{2}{|l|}{ Implant type } \\
\hline Mentor implant & $11 / 25(44)$ \\
\hline Bellagel implant & $10 / 25(40)$ \\
\hline Allergan implant & $4 / 25(16)$ \\
\hline \multicolumn{2}{|l|}{ ADM use } \\
\hline $\operatorname{ADM}(+)$ & 23/30 (77) \\
\hline $\operatorname{ADM}(-)$ & $7 / 30(23)$ \\
\hline
\end{tabular}

Values are presented as average (range) or number/number (\%). BMI, body mass index; ADM, acellular dermal matrix.

another patient had diabetes; in both patients, the conditions were managed with medication and well controlled. No patient had a history of smoking or other peripheral vessel diseases. The demographic data of all patients are summarized in Table 1.

Among the 29 women who underwent NSM (30 NSM procedures; 28 unilateral and 1 bilateral), 25 procedures involved directto implant reconstruction and five involved tissue expander reconstruction. A total of eight cases developed partial or total necrosis of the NAC (26.67\%; 8 of 30$)$. In five of those cases, surgical debridement was performed within 1 month (16.67\%; 5 of 30). The other three cases had partial necrosis that secondarily healed within 1 month without any need for surgical debridement (10\%; 3 of 30). Moreover, there was one case of mastectomy skin flap necrosis along the incision line, which was managed with debridement and revision within 1 month.

There were three cases of local infection at the operative site that
Table 2. Nipple perfusion rate for predicting NAC necrosis in immediate implant-based reconstructions

\begin{tabular}{lccc}
\hline Perfusion rate & NAC necrosis (-) & NAC necrosis (+) & Total \\
\hline$>13 \%$ & 21 & 1 & 22 \\
$\leq 13 \%$ & 1 & 7 & 8 \\
Total & 22 & 8 & 30 \\
\hline
\end{tabular}

Sensitivity: $7 /(7+1)=87.5 \%$, specificity: $21 /(21+1)=95.5 \%$, positive predictive value: $7 /(7+1)=87.5 \%$, negative predictive value: $21 /(21+1)=95.5 \%$. Fisher exact test 2 -sided $P$-value $<0.0001$.

NAC, nipple-areola complex.

Table 3. Nipple perfusion rate for predicting NAC necrosis requiring surgical debridement and revision in immediate implant-based reconstructions

\begin{tabular}{lccc}
\hline Perfusion rate & Revision (-) & Revision (+) & Total \\
\hline$>10 \%$ & 25 & 1 & 26 \\
$\leq 10 \%$ & 0 & 4 & 4 \\
Total & 25 & 5 & 30 \\
\hline
\end{tabular}

Sensitivity: $4 /(4+1)=80.0 \%$, specificity: $25 /(25+0)=100 \%$, positive predictive value: $4 /(4+0)=100 \%$, negative predictive value: $25 /(25+1)=96.2 \%$. Fisher exact test 2-sided $\mathrm{P}$-value $<0.0001$.

NAC, nipple-areola complex.

Table 4. Nipple perfusion rate for predicting NAC necrosis in directto-implant reconstructions

\begin{tabular}{lccc}
\hline Perfusion rate & NAC necrosis (-) & NAC necrosis (+) & Total \\
\hline$>13 \%$ & 18 & 1 & 19 \\
$\leq 13 \%$ & 1 & 5 & 6 \\
Total & 19 & 6 & 25 \\
\hline
\end{tabular}

Sensitivity: $5 /(5+1)=83.3 \%$, specificity: $18 /(18+1)=94.7 \%$, positive predictive value: $5 /(5+1)=83.3 \%$, negative predictive value: $18 /(18+1)=94.7 \%$. Fisher exact test 2-sided P-value $=0.001$.

NAC, nipple-areola complex.

Table 5. Nipple perfusion rate for predicting NAC necrosis requiring surgical debridement and revision in direct-to-implant reconstructions

\begin{tabular}{lccc}
\hline Perfusion rate & Revision (-) & Revision $(+)$ & Total \\
\hline$>13 \%$ & 21 & 1 & 22 \\
$\leq 13 \%$ & 0 & 3 & 3 \\
Total & 21 & 4 & 25
\end{tabular}

Sensitivity: $3 /(3+1)=75.0 \%$, specificity: $21 /(21+0)=100 \%$, positive predictive value: $3 /(3+0)=100 \%$, negative predictive value: $21 /(21+1)=95.5 \%$. Fisher exact test 2-sided $\mathrm{P}$-value $=0.002$.

NAC, nipple-areola complex.

were not related to NAC necrosis. There was no case of delayed identification of NAC necrosis at 1 month, as no patient had necrosis at 5 days postoperatively. The mean calculated nipple perfu- 

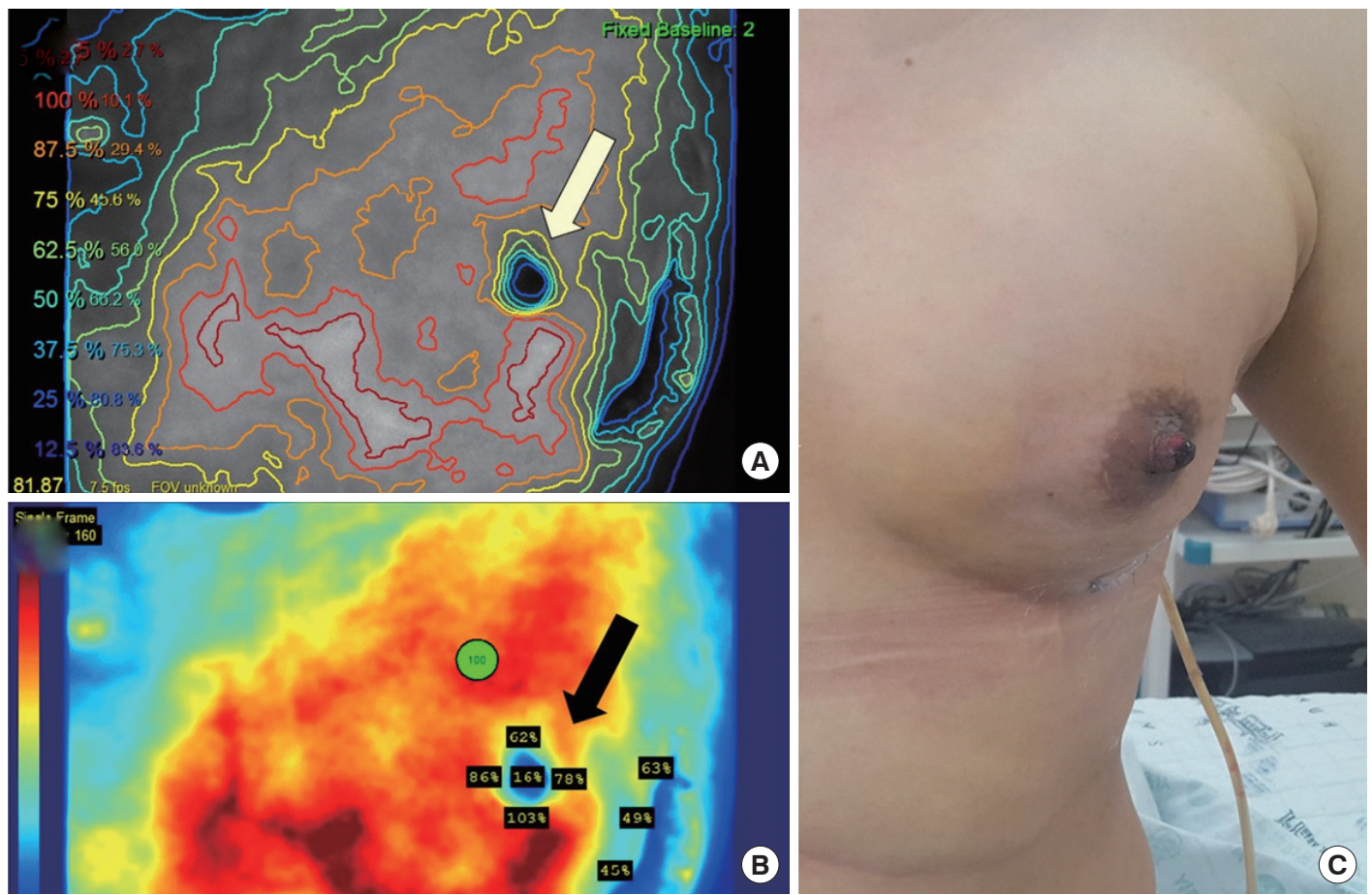

Fig. 2. Case A. A 37-year-old woman underwent nipple-sparing mastectomy and immediate implant insertion on her left breast. (A) Relative nipple perfusion (white arrow) was measured in the plateau phase. (B) The calibrated perfusion rate at the nipple center point (black arrow) was $12.7 \%$. (C) On postoperative day 5, partial-thickness necrosis was observed on her left nipple.
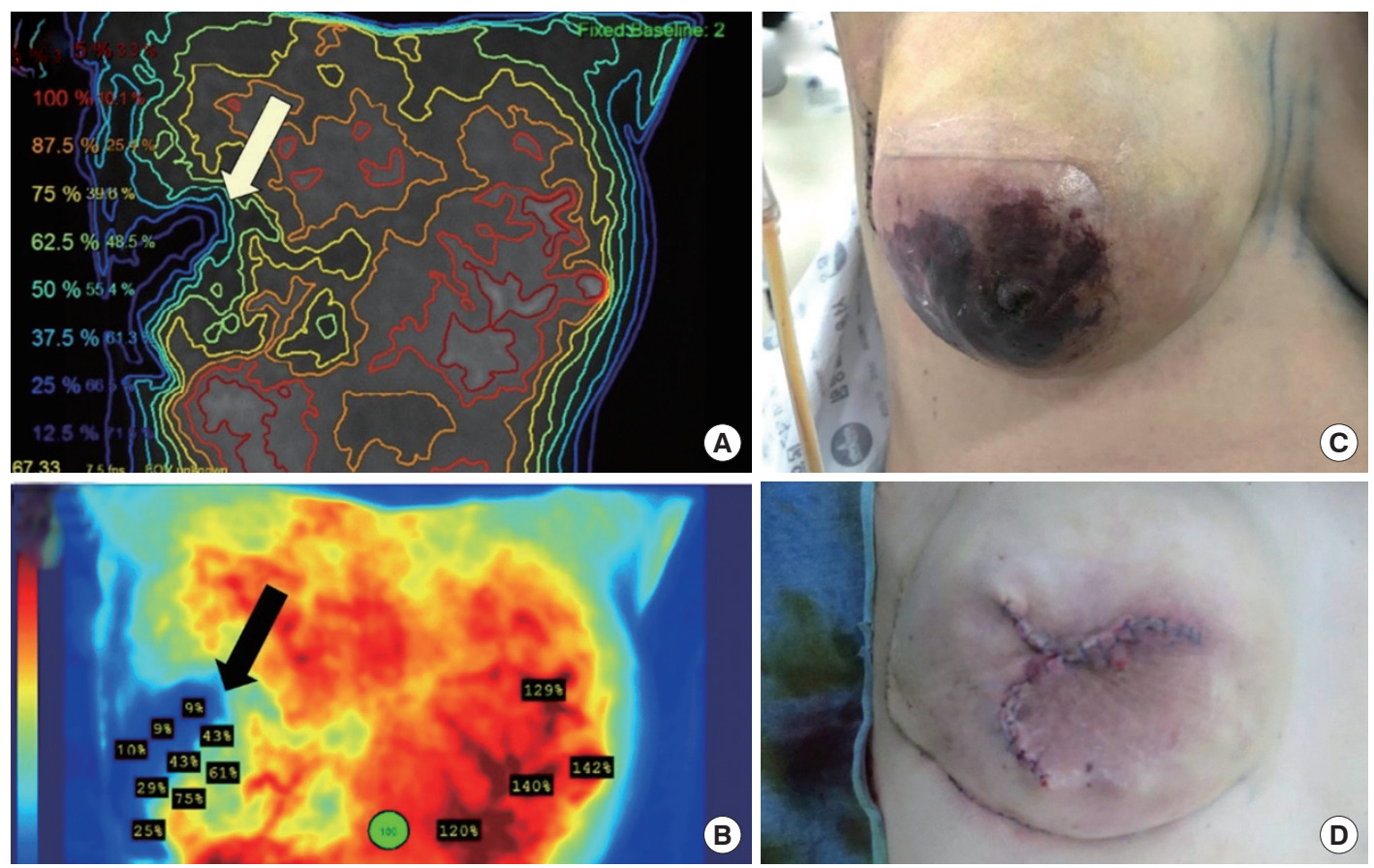

Fig. 3. Case B. A 47-year-old woman underwent nipple-sparing mastectomy and immediate implant insertion on her right breast. (A) Relative nipple perfusion (white arrow) was measured in the plateau phase. (B) The calibrated perfusion rate at the nipple center point (black arrow) was $6.42 \%$. (C) On postoperative day 5, the nipple developed total necrosis. (D) After close observation at the outpatient clinic, the patient was re-admitted on postoperative day 15 , and debridement with nipple resection was performed. 
sion rate was $26.8 \%$ for all cases, $13.8 \%$ for the NAC necrosis cases, and $8.18 \%$ for the NAC necrosis cases that required revision. Eight of the nine cases with a perfusion rate $<13 \%$ developed NAC necrosis (Fisher exact test $\mathrm{P}<0.001$ ) (Table 2), and of particular note, all four cases with a perfusion rate $<10 \%$ required partial surgical debridement or NAC excision (Fisher exact test $\mathrm{P}<0.001$ ) (Table 3). A false-positive reading for predicting necrosis occurred in one patient, whereas none of the patients had a false-positive reading for predicting revision. Moreover, a false-negative reading occurred for predicting both necrosis and revision in one patient. Tables 4 and 5 show an analysis of NAC necrosis limited only to direct-toimplant cases.

There was no statistically significant relationship between obesity $\left(\mathrm{BMI}>25 \mathrm{~kg} / \mathrm{m}^{2}\right)$ and the incidence of NAC necrosis (Fisher exact test [2-sided] $\mathrm{P}=0.3$ ). It was difficult to compare intergroup relationships between NAC necrosis and neoadjuvant chemotherapy, hypertension, diabetes mellitus, smoking, and other peripheral vascular diseases due to the small number of patients with these factors. The use of ADM in breast reconstruction showed no statistically significant relationship with NAC necrosis (Fisher exact test [2-sided] $\mathrm{P}=0.06)$.

Two representative cases of NAC necrosis are described as follows: case A developed partial NAC necrosis that secondarily healed within 1 month of wound management (Fig. 2); and case B developed total NAC necrosis requiring NAC excision as a revision operation (Fig. 3).

\section{DISCUSSION}

NSM is cosmetically superior because the patient's natural NAC is preserved and immediate direct-to-implant reconstruction, a onestage reconstructive procedure, can be considered. However, there is a relatively high incidence of NAC or incision site skin flap necrosis that may not be fully managed by meticulous dressing and secondary healing; such cases may require a revision operation and may take more time to completely heal. Moreover, this situation is difficult because it not only results in cosmetic damage of the breast contour and NAC, but also delays adjuvant chemotherapy and/or radiation therapy, which should be immediately started after the wound healing process.

If surgeons could predict the incidence of NAC after NSM more accurately through intraoperative laser-assisted angiography, the surgeon could consider tissue expander insertion rather than direct-to-implant reconstruction as an easier and less damaging management strategy in patients at a high risk of developing necrosis, thereby increasing the overall cosmesis of two-stage reconstruction. Furthermore, if the risk of NAC necrosis is moderate, and it only affects the superficial skin and partial area of NAC, surgeons may consider a topical medication, such as nitroglycerin ointment, which is well known for increasing skin flap circulation and promoting skin flap survival [7]. Moreover, for high-risk patients, the surgeon may decide that the patient requires longer hospitalization or close monitoring during follow-up at the outpatient clinic for intensive evaluation and wound care.

In our results, patients with a nipple perfusion rate $<10 \%$ were at high risk for developing NAC necrosis requiring surgical debridement and revision within 1 month. Thus, surgeons should consider a two-stage reconstruction procedure in which the first step is tissue expander insertion. Moreover, the total area of NAC necrosis was highly predictable when using the intraoperative ICG perfusion mapping image as a reference, meaning that NAC excision during immediate reconstruction surgery can be considered. In our case $\mathrm{B}$, if the surgeon had decided to insert a tissue expander with or without NAC excision, the patient's concerns and emotional stress after surgery would have been lower and a higher overall cosmetic outcome would have been obtained after the final stage of reconstruction surgery.

Patients with a nipple perfusion rate $>10 \%$ but $<13 \%$ were at a high risk for partial NAC necrosis. Thus, meticulous wound evaluation and management are needed with close follow-up monitoring, or for some conservative surgeons and patients, tissue expander insertion can be considered. In case $\mathrm{B}$, fortunately, the patient underwent tissue expander insertion according to the surgeon's decision, and the necrosis secondarily healed within 1 month. If a surgeon predicts that a patient is at a high risk of developing necrosis, whether partial or superficial, nitroglycerin ointment application could be started immediately postoperatively, and thorough wound management is essential to reduce the time and sequalae of secondary healing.

Most of the cases in this study were direct-to-implant breast reconstruction. The decision of to use an expander or implant is based on the surgeon's clinical experience, but there is nonetheless an increasing trend for direct-to-implant reconstruction at our institution. All five expander cases underwent the procedure before March 2018, and all operations performed subsequently were direct-to-implant procedures. We could not identify a statistically significant difference in NAC necrosis between these two groups due to the small number of expander cases, but the circumstances relating to mastectomy skin flaps seem to have been similar between the groups. For expander insertion reconstruction, the surgeon performs an initial inflation to obtain appropriate volume and tension, and because this procedure is not similar to total mastectomies wherein a large amount of skin is excised, the size of the expander after the initial inflation is almost the same as the final expansion state, which is also similar to the size of the implants used in direct-to-implant reconstructions. However, if the size of an expander decreases even slightly, the skin tension around the NAC is reduced, and this slight change might have an effect on NAC necrosis. In fact, we additionally analyzed the results for only the 25 direct-to-implant cases, with the same nipple perfusion rate 
cutoff value, but the statistical results were similar (Tables 4 and 5).

There were no statistically meaningful data for several patientrelated factors, such as neoadjuvant chemotherapy, diabetic mellitus, hypertension, smoking, and peripheral vascular disease, due to the small number of cases with these factors. Likewise, no statistically significant relationships were found for age and obesity, which may be attributed to the small number of total cases. In 2012, Phillips et al. [8] investigated intraoperative perfusion techniques for predicting mastectomy skin flap necrosis in association with various factors, and showed that active smoking, obesity (BMI > $30 \mathrm{~kg} /$ $\mathrm{m}^{2}$ ), large breast weight ( $>1,000 \mathrm{~g}$ ), and expander size were associated with mastectomy skin necrosis. Even though ethnic differences may exist, it is reasonable to infer that mastectomy skin necrosis and NAC necrosis share similar risk factors. A further study involving more cases with various factors, including those mentioned above, is warranted to determine more comprehensively the risk factors for NAC necrosis.

In our study, the ICG injection and video recording protocols were based on those used in other studies investigating the ability of SPY angiography to predict mastectomy skin flap necrosis. No remarkable difference was found when compared to relative perfusion mapping, but measuring the relative perfusion rate, instead of the absolute perfusion rate, might have influenced our findings [2,9].

$\mathrm{ADM}$ use was not associated with NAC necrosis. ADM is commonly used in other reconstructive procedures, as well as in breast reconstruction. Many studies have presented the potential complications of ADM use, and most surgeons think that ADM does not increase the complication rates in breast reconstruction procedures. We also believe this to be the case, especially relating to NAC necrosis [10].

The rate of necrosis as a complication in this study was $26.67 \%$ for NAC necrosis and $16.67 \%$ for necrosis requiring revision. These rates are quite high and troublesome for reconstruction sur- gery, even though this type of complication has been reported to be common in previous studies and patients received a thorough explanation before surgery that necrosis can occur because the procedures were primarily oncologic, not cosmetic, surgery. Nonetheless, the ability to predict NAC necrosis more accurately and to reduce the necrosis rate seemed important and helpful for patients.

The major limitation of this study is its small sample size and retrospective study design. Moreover, we could not precisely compare the expander insertion and direct-to implant cases. Furthermore, we did not examine the long-term sequalae of necrosis owing to the short follow-up period; hence, we may have missed some important clinical results. Moreover, a further prospective study should be followed to validate our cutoff values of $10 \%$ and $13 \%$, as these cutoff values were set based on our retrospective data, not based on scientifically-determined reference values. According to previous studies, the perfusion rates associated with necrosis vary considerably, from $<7 \%$ to $33 \%$, depending on the ICG injection protocol, imaging setting, and method of analysis, and no standard protocol has yet been established. Thus, further research is warranted [11].

The significance of this study for treatment strategies is that we were able to predict NAC necrosis during surgery relatively precisely by simply using a single quantitative value obtained from the SPY system; hence, the reconstruction plan can be changed accordingly. We suggest some algorithms based on our experiences (Fig. 4). Patients with a nipple perfusion rate $<10 \%$ would be categorized as a high-risk group, requiring NAC excision and immediate expander insertion before immediate reconstruction. Patients with a nipple perfusion rate between $10 \%$ and $13 \%$ would be categorized as a moderate-risk group, requiring intensive postoperative wound management with close follow-up after immediate reconstruction.

In conclusion, if nipple necrosis is anticipated based on a SPY

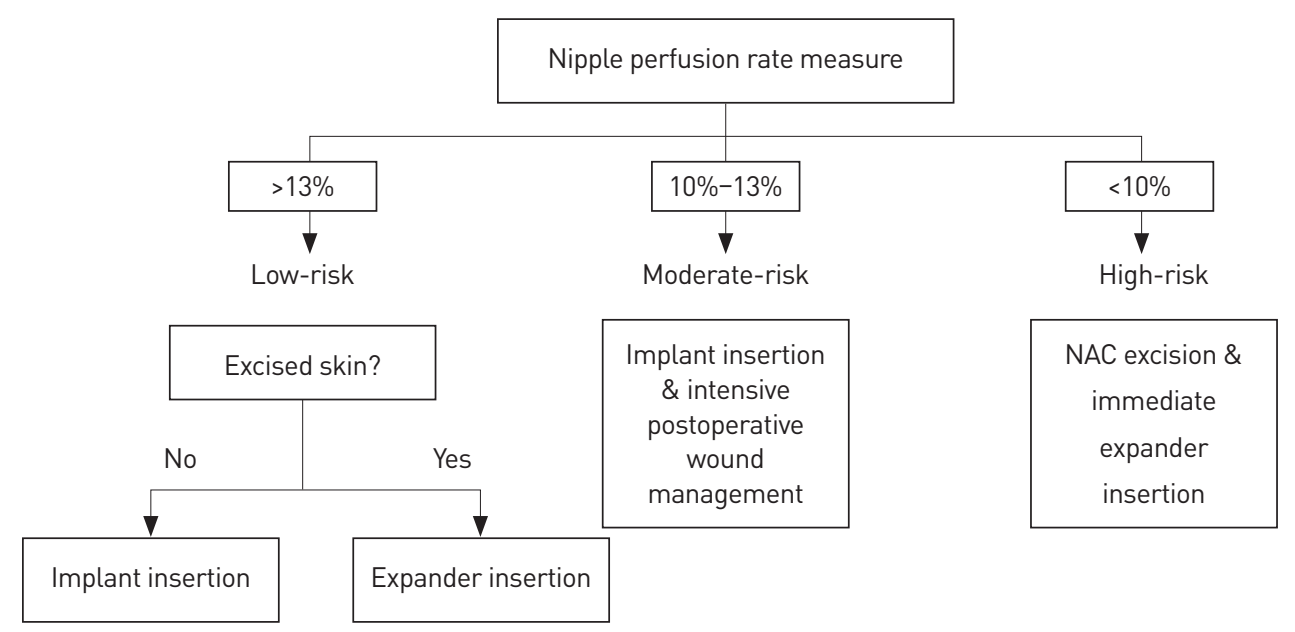

Fig. 4. The authors' recommended treatment algorithm for immediate breast reconstruction after nipple-sparing mastectomy. NAC, nippleareolar complex. 
evaluation, tissue expander insertion should be considered, rather than direct implant insertion, for better postoperative results. For moderate-risk patients, close follow-up monitoring with thorough wound management should be performed to reduce complications. Further research involving more cases might lay the groundwork for a treatment guideline for patients undergoing NSM and immediate implant-based reconstruction based on the results of a SPY perfusion evaluation.

\section{CONFLICT OF INTEREST}

No potential conflict of interest relevant to this article was reported.

\section{ETHICAL APPROVAL}

The study was approved by the Institutional Review Board of Seoul National University (IRB No. 1710-065-894) and performed in accordance with the principles of the Declaration of Helsinki.

\section{PATIENT CONSENT}

The patients provided written informed consent for the publication and the use of their images.

\section{ORCID}

Jaewoo Kim (https://orcid.org/0000-0003-2661-920X)

Il-Kug Kim (https://orcid.org/0000-0002-2428-8403)

Hak Chang (https://orcid.org/0000-0002-9888-1787)

\section{REFERENCES}

1. Gurtner GC, Jones GE, Neligan PC, et al. Intraoperative laser angiography using the SPY system: review of the literature and recommen- dations for use. Ann Surg Innov Res 2013;7:1.

2. Komorowska-Timek E, Gurtner GC. Intraoperative perfusion mapping with laser-assisted indocyanine green imaging can predict and prevent complications in immediate breast reconstruction. Plast Reconstr Surg 2010;125:1065-73.

3. Chattha $A$, Bucknor $A$, Chen $A D$, et al. Indocyanine green angiography use in breast reconstruction: a national analysis of outcomes and cost in 110,320 patients. Plast Reconstr Surg 2018;141:825-32.

4. Mattison GL, Lewis PG, Gupta SC, et al. SPY imaging use in postmastectomy breast reconstruction patients: preventative or overly conservative? Plast Reconstr Surg 2016;138:15e-21e.

5. Colwell AS, Christensen JM. Nipple-sparing mastectomy and directto-implant breast reconstruction. Plast Reconstr Surg 2017;140(5S Advances in Breast Reconstruction):44S-50S.

6. Wapnir I, Dua M, Kieryn A, et al. Intraoperative imaging of nipple perfusion patterns and ischemic complications in nipple-sparing mastectomies. Ann Surg Oncol 2014;21:100-6.

7. Yun MH, Yoon ES, Lee BI, et al. The effect of low-dose nitroglycerin ointment on skin flap necrosis in breast reconstruction after skin-sparing or nipple-sparing mastectomy. Arch Plast Surg 2017;44:509-15.

8. Phillips BT, Lanier ST, Conkling N, et al. Intraoperative perfusion techniques can accurately predict mastectomy skin flap necrosis in breast reconstruction: results of a prospective trial. Plast Reconstr Surg 2012;129:778e-788e.

9. Phillips BT, Fourman MS, Rivara A, et al. Comparing quantitative values of two generations of laser-assisted indocyanine green dye angiography systems: can we predict necrosis? Eplasty 2014;14:e44.

10. Ibrahim AM, Koolen PG, Ashraf AA, et al. Acellular dermal matrix in reconstructive breast surgery: survey of current practice among plastic surgeons. Plast Reconstr Surg Glob Open 2015;3:e381.

11. Venturi ML, Mesbahi AN, Copeland-Halperin LR, et al. SPY Elite's ability to predict nipple necrosis in nipple-sparing mastectomy and immediate tissue expander reconstruction. Plast Reconstr Surg Glob Open 2017;5:e1334. 